TRANSACTIONS OF THE

AMERICAN MATHEMATICAL SOCIETY

Volume 362, Number 10, October 2010, Pages 5107-5123

S 0002-9947(10)04965-2

Article electronically published on May 10, 2010

\title{
GENERALIZED MOSER LEMMA
}

\author{
MATHIEU STIÉNON
}

\begin{abstract}
We show how the classical Moser lemma from symplectic geometry extends to generalized complex structures (GCS) on arbitrary Courant algebroids. For this, we extend the notion of a Lie derivative to sections of the tensor bundle $\left(\otimes^{i} E\right) \otimes\left(\otimes^{j} E^{*}\right)$ with respect to sections of the Courant algebroid $E$ using the Dorfman bracket. We then give a cohomological interpretation of the existence of one-parameter families of GCS on $E$ and of flows of automorphims of $E$ identifying all GCS of such a family. In the particular case of symplectic manifolds, we recover the results of Moser. Finally, we give a criterion to detect the local triviality of arbitrary GCS which generalizes the Darboux-Weinstein theorem.
\end{abstract}

\section{Introduction}

The classical Moser lemma for symplectic manifolds 24] describes a cohomological condition for two symplectic structures to be equivalent. It can be stated as follows. For two symplectic forms $\Omega_{0}$ and $\Omega_{1}$ on a compact symplectic manifold $M$, if there exists a smooth one-parameter family $\omega_{t}$ of symplectic forms on $M$, all with the same periods and such that $\Omega_{0}=\omega_{0}$ and $\Omega_{1}=\omega_{1}$, then there is a global diffeomorphism $\varphi$ of $M$, diffeotopic to the identity and such that $\Omega_{1}=\varphi^{*} \Omega_{0}$.

Recently there has been increasing interest in generalized complex structures 2, 4, 5, 7, 8, 9, 10, 13, 14, 15, 16, which comprise both symplectic and complex structures as special cases. It is natural to ask if Moser's lemma extends to generalized complex geometry. The aim of the present paper is to give an affirmative answer to this question. While up to now, most of the research on generalized complex structures focused on exact Courant algebroids $T M \oplus T^{*} M$ [28, in this paper, we work on generic Courant algebroids. A generalized complex structure on a Courant algebroid $E$ is a bundle map $J: E \rightarrow E$ satisfying $J^{2}=-1$, which is orthogonal with respect to the symmetric pairing and whose Nijenhuis torsion vanishes 3 .

As is well known, the proof of the classical Moser lemma involves the Lie derivative of symplectic forms with respect to a vector field. Here, as a first step, we introduce a concept of a Lie derivative of generalized complex strutures with respect to sections of a Courant algebroid. Indeed, we introduce the Lie derivative

Received by the editors April 4, 2008.

2010 Mathematics Subject Classification. Primary 53C15, 17B62, $17 \mathrm{~B} 66$.

Key words and phrases. Courant algebroid, Moser lemma, generalized complex structure, Dorfman bracket, symplectic form.

This work was supported by the European Union through the FP6 Marie Curie RTN ENIGMA (Contract number MRTN-CT-2004-5652) and by the E.S.I. Vienna through a Junior Research Fellowship.

(C)2010 American Mathematical Society Reverts to public domain 28 years from publication 5107 
of sections of the tensor bundle $\left(\otimes^{i} E\right) \otimes\left(\otimes^{j} E^{*}\right)$ with respect to sections of the Courant algebroid $E$. We hope this construction will be of independent interest in the future. Such a Lie derivative can be defined exactly as in the classical case. Namely, via the Dorfman bracket, one can think of $\Gamma(E)$ as a subset of $\mathfrak{a u t}(E)$, the infinitesimal automorphisms of the Courant algebroid $E$. Thus any section of $E$ generates a local flow of automorphims of the Courant algebroid $E$. We define the Lie derivative of any section of $\left(\otimes^{i} E\right) \otimes\left(\otimes^{j} E^{*}\right)$ as the time-derivative at $t=0$ of the pull-back of this section by the flow. For any $X, Y \in \Gamma(E)$, the Lie derivative $\mathcal{L}_{X} Y$ is simply the Dorfman bracket $X \circ Y$. The Lie derivative of a bundle map $J: E \rightarrow E$, seen as a section of $E^{*} \otimes E$, is given by $\left(\mathcal{L}_{X} J\right)(Y)=\mathcal{L}_{X} J(Y)-J\left(\mathcal{L}_{X} Y\right)$, $\forall X, Y \in \Gamma(E)$. This formula reduces exactly to the usual Lie derivative of the symplectic form when $J$ corresponds to a symplectic structure and $X$ is a vector field.

With the help of the Lie derivative, we are able to translate the infinitesimal isomorphism condition for $J_{t}$,

$$
\dot{J}_{t}+\mathcal{L}_{x_{t}} J_{t}=0, \quad \forall t \text { with } x_{t} \in \Gamma(E),
$$

as the exactness, at every time $t$, of a family $t \rightarrow \dot{\omega}_{t}$ of Lie algebroid 2-cocycles relative to the one-parameter family of Lie algebroids $t \mapsto L_{t}$, where $L_{t}$ is the $+i$ eigenbundle of $J_{t}$. Hence we recover exactly the same situation as in the classical Moser lemma context [24].

As first examples, we consider symplectic and complex structures on a Lie algebroid $A$. The Courant algebroid $E$ involved is the double $A \oplus A^{*}$ of $A$. When $A$ is the tangent bundle Lie algebroid $T M$, we recover the usual Moser lemma for symplectic manifolds and the classical result of Kodaira 19], respectively. Other examples of generalized complex structures are given by Hamiltonian operators in the sense of Liu-Weinstein-Xu [22]. We describe conditions when such operators induce isomorphic generalized complex structures. Holomorphic Poisson structures are a special case.

As another application, we give a Darboux-Weinstein style theorem for generalized complex strutures. More precisely, we describe local cohomological conditions which guarantee the local triviality of a generalized complex structure on the standard Courant algebroid. This cohomological condition is always satisfied for symplectic manifolds. On the other hand, for an integrable complex structure, this condition should be related to Kohn's proof [20] of the Newlander-Nirenberg theorem [26].

\section{LIE DERIVATIVES}

The symmetry groups of generalized Courant algebroids were first studied by Roytenberg from the point of view of supergeometry [27]. In this section, we recall some basic ingredients which are needed later on. Here we choose the more conventional description of symmetries in terms of linear vector fields on Courant algebroids, which better fits our needs. See also [17, 18, [5] for discussions on a similar topic.

Definition 2.1 ([22]). A Courant algebroid is a triple consisting of a vector bundle $E \rightarrow M$ equipped with a nondegenerate symmetric bilinear form $\langle\cdot, \cdot\rangle$, a skewsymmetric bracket $\llbracket \cdot, \cdot \rrbracket$ on $\Gamma(E)$, and a smooth bundle map $E \stackrel{\rho}{\rightarrow} T M$ called the anchor. These induce a natural differential operator $\mathcal{D}: C^{\infty}(M) \rightarrow \Gamma(E)$ defined 
by

$$
\langle\mathcal{D} f, a\rangle=\frac{1}{2} \rho(a) f
$$

for all $f \in C^{\infty}(M)$ and $a \in \Gamma(E)$.

These structures must be compatible in the following sense: $\forall a, b, c \in \Gamma(E)$ and $\forall f, g \in C^{\infty}(M)$,

$$
\begin{aligned}
& \rho(\llbracket a, b \rrbracket)=[\rho(a), \rho(b)], \\
& \llbracket \llbracket a, b \rrbracket, c \rrbracket+\llbracket \llbracket b, c \rrbracket, a \rrbracket+\llbracket \llbracket c, a \rrbracket, b \rrbracket=\frac{1}{3} \mathcal{D}(\langle\llbracket a, b \rrbracket, c\rangle+\langle\llbracket b, c \rrbracket, a\rangle+\langle\llbracket c, a \rrbracket, b\rangle), \\
& \llbracket a, f b \rrbracket=f \llbracket a, b \rrbracket+(\rho(a) f) b-\langle a, b\rangle \mathcal{D} f, \\
& \rho \circ \mathcal{D}=0, \text { i.e. }\langle\mathcal{D} f, \mathcal{D} g\rangle=0, \\
& \rho(a)\langle b, c\rangle=\langle\llbracket a, b \rrbracket+\mathcal{D}\langle a, b\rangle, c\rangle+\langle b, \llbracket a, c \rrbracket+\mathcal{D}\langle a, c\rangle\rangle .
\end{aligned}
$$

The main example is due to T. Courant [1]. Given a smooth manifold $M$, the bundle $T M \oplus T^{*} M \rightarrow M$ carries a natural Courant algebroid structure, where the anchor is the projection onto the tangent component and the pairing and bracket are given, respectively, by

$$
\begin{gathered}
\langle X+\xi, Y+\eta\rangle=\frac{1}{2}(\xi(Y)+\eta(X)) \\
\llbracket X+\xi, Y+\eta \rrbracket=[X, Y]+L_{X} \eta-L_{Y} \xi+\frac{1}{2} d(\xi(Y)-\eta(X)),
\end{gathered}
$$

$\forall X, Y \in \mathfrak{X}(M), \forall \xi, \eta \in \Omega^{1}(M)$.

The nonsymmetric law

$$
a \circ b:=\llbracket a, b \rrbracket+\mathcal{D}\langle a, b\rangle
$$

is called the Dorfman bracket [27]. The following result is due to Roytenberg [27].

Proposition 2.2. For all $a, b, c \in \Gamma(E)$ and $f \in C^{\infty}(M)$, one has:

$$
\begin{array}{ll}
\mathcal{D} f \circ a=0, & a \circ \llbracket b, c \rrbracket=\llbracket a \circ b, c \rrbracket+\llbracket b, a \circ c \rrbracket, \\
a \circ(f b)=f(a \circ b)+(\rho(a) f) b, & \rho(a)\langle b, c\rangle=\langle a \circ b, c\rangle+\langle b, a \circ c\rangle .
\end{array}
$$

Any section of the dual bundle $E^{*} \rightarrow M$ can be seen as a fiberwise linear function on $E$ and vice versa. In other words, $\Gamma\left(E^{*}\right)$ is naturally a subspace of $C^{\infty}(E)$. Recall that an infinitesimal automorphism of a vector bundle $E \stackrel{\pi}{\rightarrow} M$ corresponds exactly to a vector field on $E$ (i.e., a derivation of $C^{\infty}(E)$ ) under which the subspaces $\pi^{*} C^{\infty}(M)$ and $\Gamma\left(E^{*}\right)$ are stable [23. Proposition 2.2]. The latter is equivalent to a covariant differential operator on $E^{*}$ [23], i.e. a pair of differential operators $\delta^{0}: C^{\infty}(M) \rightarrow C^{\infty}(M)$ and $\delta^{1}: \Gamma\left(E^{*}\right) \rightarrow \Gamma\left(E^{*}\right)$ satisfying

$$
\begin{array}{ll}
\delta^{0}(f g)=f \delta^{0}(g)+\delta^{0}(f) g, & \forall f, g \in C^{\infty}(M), \\
\delta^{1}(f \alpha)=f \delta^{1}(\alpha)+\delta^{0}(f) \alpha, & \forall \alpha \in \Gamma\left(E^{*}\right), \forall f \in C^{\infty}(M) .
\end{array}
$$

From now on, we assume that $E$ is a Courant algebroid. By $\mathfrak{a u t}(E)$, we denote the Lie algebra of infinitesimal automorphisms of the Courant algebroid $E$. The symmetric pairing $\langle\cdot, \cdot\rangle$ identifies $\Gamma(E)$ with $\Gamma\left(E^{*}\right)$ by $x \mapsto\langle x, \cdot\rangle$. Therefore, one easily recovers the following theorem of Roytenberg [27], which he proved using super-geometry. 
Proposition 2.3. The Lie algebra aut $(E)$ consists of those covariant differential operators $\delta=\left(\delta^{0}, \delta^{1}\right)$ on $E$, where $\delta^{0}: C^{\infty}(M) \rightarrow C^{\infty}(M)$ and $\delta^{1}: \Gamma(E) \rightarrow \Gamma(E)$, satisfying the additional properties:

$$
\delta^{0}\langle x, y\rangle=\left\langle\delta^{1} x, y\right\rangle+\left\langle x, \delta^{1} y\right\rangle
$$

and

$$
\delta^{1} \llbracket x, y \rrbracket=\llbracket \delta^{1} x, y \rrbracket+\llbracket x, \delta^{1} y \rrbracket,
$$

for all $x, y \in \Gamma(E)$.

For any $z \in \Gamma(E)$, define

$$
\delta_{z}^{0}: C^{\infty}(M) \rightarrow C^{\infty}(M) \quad \text { and } \quad \delta_{z}^{1}: \Gamma(E) \rightarrow \Gamma(E)
$$

by

$$
\delta_{z}^{0}(f)=\rho(z)(f) \quad \text { and } \quad \delta_{z}^{1}(x)=z \circ x, \quad \forall f \in C^{\infty}(M), \forall x \in \Gamma(E) .
$$

It follows from Propositions 2.2 and 2.3 that $\delta_{z}=\left(\delta_{z}^{0}, \delta_{z}^{1}\right)$ is an infinitesimal automorphism of the Courant algebroid $E$, i.e. $\delta_{z} \in \mathfrak{a u t}(E)$.

By $\phi_{t}$ we denote the (local) flow

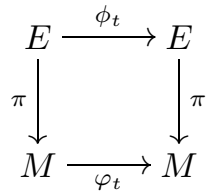

generated by the vector field on $E$ corresponding to $\delta_{z}$. The one-parameter group $t \mapsto \phi_{t}$ acts on the space of sections of $E \rightarrow M$ by

$$
\phi_{t}^{*}: \Gamma(E) \rightarrow \Gamma(E): \sigma \mapsto \phi_{t}^{-1} \circ \sigma \circ \varphi_{t} .
$$

By abuse of notation, we use the same symbol $\phi_{t}$ (resp. $\left.\phi_{t}^{*}\right)$ to denote the induced flow on the tensor bundles $E_{j}^{i}=\left(\otimes^{i} E\right) \otimes\left(\otimes^{j} E^{*}\right)(i, j \in\{0,1,2, \ldots\})$ (resp. the induced action on the spaces of sections of the $E_{j}^{i}$ 's).

For any section $\sigma \in \Gamma\left(E_{j}^{i}\right)$, define $\mathcal{L}_{z} \sigma \in \Gamma\left(E_{j}^{i}\right)$ by

$$
\mathcal{L}_{z} \sigma=\left.\frac{d}{d \tau} \phi_{\tau}^{*} \sigma\right|_{\tau=0}
$$

(see [1, Theorem 2.2.20]). Thus we have the usual identity:

$$
\left.\frac{d}{d \tau} \phi_{\tau}^{*} \sigma\right|_{\tau=t}=\phi_{t}^{*}\left(\mathcal{L}_{z} \sigma\right)
$$

In the following proposition, we give a list of important properties of this Lie derivative, which will be useful in future discussions.

Proposition 2.4. For all $f, g \in C^{\infty}(M)$ and $x, y, z \in \Gamma(E)$, we have:

$$
\begin{aligned}
& \mathcal{L}_{z} f=\rho(z) f, \quad \mathcal{L}_{z}\langle x, y\rangle=\left\langle\mathcal{L}_{z} x, y\right\rangle+\left\langle x, \mathcal{L}_{z} y\right\rangle, \\
& \mathcal{L}_{z} x=z \circ x, \quad \mathcal{L}_{z} \llbracket x, y \rrbracket=\llbracket \mathcal{L}_{z} x, y \rrbracket+\llbracket x, \mathcal{L}_{z} y \rrbracket, \\
& \mathcal{L}_{\mathcal{D} f} x=0, \quad \mathcal{L}_{f x} y=f \mathcal{L}_{x} y-\left(\mathcal{L}_{y} f\right) x+2\langle x, y\rangle \mathcal{D} f, \\
& \mathcal{L}_{\llbracket x, y \rrbracket}=\left[\mathcal{L}_{x}, \mathcal{L}_{y}\right] .
\end{aligned}
$$

Moreover,

$$
\begin{aligned}
& \mathcal{L}_{z}(\sigma \otimes \tau)=\mathcal{L}_{z} \sigma \otimes \tau+\sigma \otimes \mathcal{L}_{z} \tau \text { for all } \sigma, \tau \in \bigoplus_{i, j} E_{j}^{i} \\
& \text { and }\left[\delta, \mathcal{L}_{z}\right]=\mathcal{L}_{\delta^{1} z} \text { for all } \delta \in \mathfrak{a u t}(E) .
\end{aligned}
$$


In particular, if $J: E \rightarrow E$ is a bundle map over the identity $M \stackrel{\text { id }}{\rightarrow} M$, i.e. $J \in \Gamma\left(E^{*} \otimes E\right)$, then

$$
\left(\mathcal{L}_{x} J\right)(y)=\mathcal{L}_{x}(J y)-J\left(\mathcal{L}_{x} y\right), \quad \forall x, y \in \Gamma(E) .
$$

Example 2.5. For the standard Courant algebroid structure on $T M \oplus T^{*} M$, one has

$$
\mathcal{L}_{X+\xi}(Y+\eta)=L_{X}(Y+\eta)-i_{Y} d \xi, \quad \forall X, Y \in \mathfrak{X}(M), \forall \xi, \eta \in \Omega^{1}(M),
$$

where $L$ denotes the usual Lie derivative.

The following result appeared in [17.

Proposition 2.6. Let $E$ denote the standard Courant algebroid structure on $T M \oplus$ $T^{*} M$. Then, for all $X \in \mathfrak{X}(M)$ and $\xi \in \Omega^{1}(M)$, the one-parameter groups integrating the infinitesimal automorphisms $\delta_{X}, \delta_{\xi}, \delta_{X+\xi} \in \mathfrak{a u t}(E)$ are given respectively by

$$
\begin{aligned}
& e^{t \delta_{X}}(Y+\eta)=\left(\varphi_{t}\right)_{*} Y+\left(\varphi_{t}^{-1}\right)^{*} \eta \\
& e^{t \delta_{\xi}}(Y+\eta)=Y+\eta+t i_{Y} d \xi \\
& e^{t \delta_{X+\xi}}(Y+\eta)=\left(\varphi_{t}\right)_{*} Y+\left(\varphi_{t}^{-1}\right)^{*} \eta+\int_{0}^{t}\left(\varphi_{t-\tau}^{-1}\right)^{*}\left(i_{\varphi_{\tau *} Y} d \xi\right) d \tau,
\end{aligned}
$$

where $t \mapsto \varphi_{t}$ denotes the flow of the vector field $X$, i.e. satisfies

$$
X_{\varphi_{t}(m)}=\left.\frac{d}{d t} \varphi_{\tau}(m)\right|_{\tau=t} .
$$

In case the section $X_{t}+\xi_{t} \in \Gamma(E)$ is time-dependent, its flow

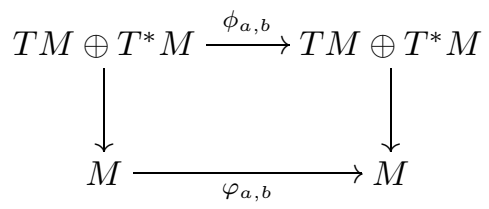

from time $a$ to $b$ is given by

$$
\phi_{a, b}(Y+\eta)=\left(\varphi_{a, b}\right)_{*} Y+\left(\varphi_{b, a}\right)^{*} \eta+\int_{a}^{b}\left(\varphi_{b, \tau}\right)^{*}\left(i_{\left(\varphi_{a, \tau}\right)_{*} Y} d \xi_{\tau}\right) d \tau,
$$

where $\varphi_{a, b}$ denotes the flow of the time-dependent vector field $X_{t}$ from time a to $b$.

\section{MAIN THEOREM}

Let $E$ be a Courant algebroid on a smooth manifold $M$ and let $J: E \rightarrow E$ be a vector bundle map over the identity such that $J^{2}=-$ id. Then the complexification $E_{\mathbb{C}}:=E \otimes \mathbb{C}$ with the extended $\mathbb{C}$-linear Courant algebroid structure decomposes as the direct sum $L \oplus \bar{L}$ of the eigenbundles of $J$. Here $L$ is associated to the eigenvalue $+i$ and its complex conjugate $\bar{L}$ to $-i$. The bundle map $J$ is called a generalized complex structure if $J$ is orthogonal with respect to $\langle\cdot, \cdot\rangle$ (this forces $L$ and $\bar{L}$ to be isotropic) and the spaces of sections $\Gamma(L)$ and $\Gamma(\bar{L})$ are closed under the Courant bracket, or equivalently, $J$ is "integrable":

$$
\llbracket J x, J y \rrbracket-\llbracket x, y \rrbracket-J(\llbracket J x, y \rrbracket+\llbracket x, J y \rrbracket)=0, \quad \forall x, y \in \Gamma(E) .
$$

We refer the reader to $2,4,4,7,8,9,10,13,14,15,16$, for more details. 
The group of automorphisms of the Courant algebroid acts on its set of generalized complex structures in a natural way.

Given a smooth family $t \mapsto J_{t}$ of generalized complex structures on $E$, we define a 2 -form $\omega_{t} \in \Gamma\left(\wedge^{2} E^{*}\right)$ by

$$
\omega_{t}(x, y)=\left\langle x, J_{t} y\right\rangle, \quad \forall x, y \in \Gamma(E) .
$$

It is clear that $\omega_{t}$ is skew-symmetric. By $L_{t}$ we denote the $+i$ eigenbundle of $J_{t}: E_{\mathbb{C}} \rightarrow E_{\mathbb{C}}$. Thus $L_{t}$ is a Dirac structure on $E_{\mathbb{C}}$. Indeed $\left(L_{t}, \overline{L_{t}}\right)$ is a (complex) Lie bialgebroid [23].

The restriction of the time derivative of $\omega_{t} \in \Gamma\left(\wedge^{2} E^{*}\right)$ to the subbundle $L_{t}$ defines a 2 -form $\dot{\omega}_{t} \in \Gamma\left(\wedge^{2} L_{t}^{*}\right)$ :

$$
\dot{\omega}_{t}(v, w)=\left\langle v, \dot{J}_{t} w\right\rangle, \quad \forall v, w \in \Gamma\left(L_{t}\right) .
$$

Remark 3.1. Differentiating $J_{t} J_{t}=-\mathrm{id}$, we obtain $\dot{J}_{t} J_{t}+J_{t} \dot{J}_{t}=0$. Therefore, the map $\dot{J}_{t}$ swaps the eigenbundles $L_{t}$ and $\overline{L_{t}}$.

The cohomology of the canonical differential complex

$$
\cdots \stackrel{d_{L_{t}}}{\longrightarrow} \Gamma\left(\wedge^{\bullet-1} L_{t}^{*}\right) \stackrel{d_{L_{t}}}{\longrightarrow} \Gamma\left(\wedge^{\bullet} L_{t}^{*}\right) \stackrel{d_{L_{t}}}{\longrightarrow} \Gamma\left(\wedge^{\bullet+1} L_{t}^{*}\right) \stackrel{d_{L_{t}}}{\longrightarrow} \cdots
$$

associated to the Lie algebroid $L_{t}$ [] is the Lie algebroid cohomology of $L_{t}$.

Proposition 3.2. $d_{L_{t}} \dot{\omega}_{t}=0, \forall t$.

Proof. A straightforward computation using Definition 2.1 and the orthogonality of $J_{t}$ gives

$$
\left(d_{L_{t}} \dot{\omega}_{t}\right)(v, w, z)=\left\langle v, \llbracket \dot{J}_{t} z, w \rrbracket+\llbracket z, \dot{J}_{t} w \rrbracket-\dot{J}_{t} \llbracket z, w \rrbracket\right\rangle \quad \forall v, w, z \in \Gamma\left(L_{t}\right) .
$$

Since $J_{t}$ is integrable $\forall t$, we have

$$
\llbracket J_{t} v, J_{t} w \rrbracket-\llbracket v, w \rrbracket-J_{t}\left(\llbracket J_{t} v, w \rrbracket+\llbracket v, J_{t} w \rrbracket\right)=0 .
$$

Differentiating w.r.t. $t$, we get

$$
\llbracket \dot{J}_{t} v, J_{t} w \rrbracket+\llbracket J_{t} v, \dot{J}_{t} w \rrbracket-\dot{J}_{t}\left(\llbracket J_{t} v, w \rrbracket+\llbracket v, J_{t} w \rrbracket\right)-J_{t}\left(\llbracket \dot{J}_{t} v, w \rrbracket+\llbracket v, \dot{J}_{t} w \rrbracket\right)=0 .
$$

Now, taking $v, w \in \Gamma\left(L_{t}\right)$, the above relation becomes

$$
i \llbracket \dot{J}_{t} v, w \rrbracket+i \llbracket v, \dot{J}_{t} w \rrbracket-2 i \dot{J}_{t} \llbracket v, w \rrbracket-J_{t}\left(\llbracket \dot{J}_{t} v, w \rrbracket+\llbracket v, \dot{J}_{t} w \rrbracket\right)=0
$$

and, multiplying by $(-i)$, we obtain

$$
\frac{1+i J_{t}}{2}\left(\llbracket \dot{J}_{t} v, w \rrbracket+\llbracket v, \dot{J}_{t} w \rrbracket\right)=\dot{J}_{t} \llbracket v, w \rrbracket .
$$

Hence, since $L_{t}$ is isotropic,

$$
\left\langle z, \llbracket \dot{J}_{t} v, w \rrbracket+\llbracket v, \dot{J}_{t} w \rrbracket-\dot{J}_{t} \llbracket v, w \rrbracket\right\rangle=0, \quad \forall v, w, z \in \Gamma\left(L_{t}\right) .
$$

The result follows from (3.1) and (3.2).

Lemma 3.3. Let $t \mapsto J_{t}(t \in[0,1])$ be a smooth family of generalized complex structures on a Courant algebroid $E$. There exists a smooth path $t \mapsto \beta_{t}$ in $\Gamma\left(L_{t}^{*}\right)$ such that

$$
\dot{\omega}_{t}=d_{L_{t}} \beta_{t}, \quad \forall t
$$

if, and only if,

$$
\dot{J}_{t}+\mathcal{L}_{x_{t}} J_{t}=0, \quad \forall t
$$

where $x_{t}=\frac{1}{2 i}\left(z_{t}-\overline{z_{t}}\right) \in \Gamma(E)$ is the imaginary part of the unique section $z_{t} \in \Gamma\left(L_{t}\right)$ such that $\beta_{t}=\left\langle\overline{z_{t}}, \cdot\right\rangle$. 
Proof. For $v, w \in \Gamma\left(L_{t}\right)$, one has $\dot{\omega}_{t}(v, w)=\left\langle v, \dot{J}_{t} w\right\rangle$ and

$$
\begin{aligned}
\left(d_{L_{t}} \beta_{t}\right)(v, w) & =\rho(v) \beta_{t}(w)-\rho(w) \beta_{t}(v)-\beta_{t}(\llbracket v, w \rrbracket) \\
& =\rho(v)\left\langle\overline{z_{t}}, w\right\rangle-\rho(w)\left\langle\overline{z_{t}}, v\right\rangle-\left\langle\overline{z_{t}}, \llbracket v, w \rrbracket\right\rangle \\
& =2\left\langle\mathcal{D}\left\langle\overline{z_{t}}, w\right\rangle, v\right\rangle-\left(\left\langle\mathcal{L}_{w} \overline{z_{t}}, v\right\rangle+\left\langle\overline{z_{t}}, \mathcal{L}_{w} v\right\rangle\right)-\left\langle\overline{z_{t}}, \llbracket v, w \rrbracket\right\rangle \\
& =2\left\langle\mathcal{D}\left\langle\overline{z_{t}}, w\right\rangle, v\right\rangle-\left\langle\llbracket w, \overline{z_{t}} \rrbracket+\mathcal{D}\left\langle w, \overline{z_{t}}\right\rangle, v\right\rangle-\left\langle\overline{z_{t}}, \llbracket w, v \rrbracket\right\rangle-\left\langle\overline{z_{t}}, \llbracket v, w \rrbracket\right\rangle \\
& =\left\langle v, \llbracket \bar{\llbracket}, w \rrbracket+\mathcal{D}\left\langle\overline{z_{t}}, w\right\rangle\right\rangle=\left\langle v, \mathcal{L}_{\overline{z_{t}}} w\right\rangle .
\end{aligned}
$$

Hence

$$
\begin{array}{ll} 
& \dot{\omega}_{t}=d_{L_{t}} \beta_{t} \\
\Leftrightarrow \quad & \left\langle v, \dot{j}_{t} w-\mathcal{L}_{\overline{z_{t}}} w\right\rangle=0, \quad \forall v \in \Gamma\left(L_{t}\right) \\
\Leftrightarrow \quad & \dot{J}_{t} w-\mathcal{L}_{\overline{z_{t}}} w \in \Gamma\left(L_{t}\right) \\
\Leftrightarrow & \left(\dot{J}_{t} w\right)_{\overline{L_{t}}}=\left(\mathcal{L}_{\overline{z_{t}}} w\right)_{\overline{L_{t}}},
\end{array}
$$

for all $w \in \Gamma\left(L_{t}\right)$. But $\dot{J}_{t} w \in \Gamma\left(\overline{L_{t}}\right)$ as $w \in \Gamma\left(L_{t}\right)$. Therefore,

$$
\dot{J}_{t} w=\left(\mathcal{L}_{\overline{z_{t}}} w\right)_{\overline{L_{t}}}, \quad \forall w \in \Gamma\left(L_{t}\right) .
$$

Since $\Gamma\left(L_{t}\right)$ is $\llbracket \cdot, \cdot \rrbracket$-closed and $L_{t}$ is $\langle\cdot, \cdot\rangle$-isotropic,

$$
\mathcal{L}_{z_{t}} w=\llbracket z_{t}, w \rrbracket+\mathcal{D}\left\langle z_{t}, w\right\rangle=\llbracket z_{t}, w \rrbracket \in \Gamma\left(L_{t}\right)
$$

and

$$
\left(\mathcal{L}_{z_{t}} w\right)_{\overline{L_{t}}}=0
$$

for all $w \in \Gamma\left(L_{t}\right)$. Subtracting (3.5) from (3.4), we get

$$
\begin{aligned}
\dot{J}_{t} w=\left(\mathcal{L}_{\left(\overline{z_{t}}-z_{t}\right)} w\right)_{\overline{L_{t}}}=-2 i\left(\mathcal{L}_{x_{t}} w\right)_{\overline{L_{t}}}=-2 i \frac{1+i J_{t}}{2}\left(\mathcal{L}_{x_{t}} w\right) \\
\quad=-\mathcal{L}_{x_{t}}(i w)+J_{t}\left(\mathcal{L}_{x_{t}} w\right)=-\mathcal{L}_{x_{t}}\left(J_{t} w\right)+J_{t}\left(\mathcal{L}_{x_{t}} w\right)=-\left(\mathcal{L}_{x_{t}} J_{t}\right)(w),
\end{aligned}
$$

for all $w \in \Gamma\left(L_{t}\right)$. Thus,

$$
\dot{J}_{t} v+\left(\mathcal{L}_{x_{t}} J_{t}\right)(v)=0, \quad \forall v \in \Gamma\left(L_{t}\right) .
$$

Since $x_{t}$ is a real section of $E$ and the endomorphisms $J_{t}$ and $\dot{J}_{t}$ are real, we also have

$$
\dot{J}_{t} \bar{v}+\left(\mathcal{L}_{x_{t}} J_{t}\right)(\bar{v})=0, \quad \forall \bar{v} \in \Gamma\left(\overline{L_{t}}\right) .
$$

Therefore, $\dot{J}_{t}+\mathcal{L}_{x_{t}} J_{t}=0$, for all $t$.

Theorem 3.4. Assume that $M$ is a compact manifold and that $E$ is a Courant algebroid over $M$. Assume that $J_{0}$ and $J_{1}$ are two generalized complex structures on $E$, which are connected by a smooth family of generalized complex structures $t \mapsto J_{t}(t \in[0,1])$ on $E$. Assume there exists a smooth path $t \mapsto \beta_{t}$ in $\Gamma\left(L_{t}^{*}\right)$ such that $\dot{\omega}_{t}=d_{L_{t}} \beta_{t}, \forall t$. Then $J_{0}$ and $J_{1}$ are isomorphic.

Proof. The idea of the proof is to construct a smooth one-parameter family of automorphisms $t \mapsto \phi_{t}$ in $\operatorname{Aut}(E)$ such that $\phi_{t} \circ J_{0}=J_{t} \circ \phi_{t}, \forall t$. Since $L_{t}^{*} \simeq \overline{L_{t}}$, there exists a unique time-dependent section $z_{t} \in \Gamma\left(L_{t}\right)$ such that $\beta_{t}=\left\langle\overline{z_{t}}, \cdot\right\rangle$. Set $x_{t}=\frac{1}{2 i}\left(z_{t}-\overline{z_{t}}\right) \in \Gamma(E)$. It follows from Lemma 3.3 that

$$
\dot{J}_{t}+\mathcal{L}_{x_{t}} J_{t}=0, \quad \forall t .
$$

Therefore

$$
\left.\frac{d}{d \tau} \phi_{\tau}^{-1} \circ J_{\tau} \circ \phi_{\tau}\right|_{\tau=t}=\phi_{t}^{-1} \circ\left(\dot{J}_{t}+\mathcal{L}_{x_{t}} J_{t}\right) \circ \phi_{t}=0,
$$


where $\phi_{t} \in \operatorname{Aut}(E)$ is the flow of $\delta_{x_{t}} \in \mathfrak{a u t}(E)$ (as in Section 2), which always exists since $M$ is compact.

Remark 3.5. When $M$ is not compact, the above theorem is still valid if $x_{t}$ is complete, i.e. if its flow exists for arbitrary time.

Remark 3.6. In the standard Courant algebroid $T M \oplus T^{*} M$ case, a remarkable deformation/obstruction theory for generalized complex manifolds was developed by Gualtieri in his thesis [13. It would be interesting to explore the connection between the theorem above and Gualtieri's deep results. Moreover, a special situation of Theorem 3.4, one where the path $J_{t}$ is constant, was considered by $\mathrm{Hu}$ in [18, Remark 3.6] using a different method.

\section{EXAMPLES}

Example 1. A symplectic form on a Lie algebroid $A \rightarrow M$ is a smooth section $\Omega$

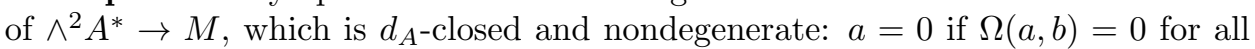
$b \in \Gamma(A)$ [25]. Here $d_{A}: \Gamma\left(\wedge^{k} A^{*}\right) \rightarrow \Gamma\left(\wedge^{k+1} A^{*}\right)$ is the Lie algebroid cohomology differential.

Let $\Omega_{0}, \Omega_{1}$ be a pair of symplectic forms on $A$, which can be connected by a smooth family $t \mapsto \Omega_{t}$ of symplectic 2 -forms. Having endowed $A^{*}$ with the trivial Lie algebroid structure, the pair $\left(A, A^{*}\right)$ becomes a Lie bialgebroid. On its "double", the Courant algebroid $E=A \oplus A^{*}$, we get the one-parameter family of generalized complex structures

$$
J_{t}=\left(\begin{array}{cc}
0 & -\pi_{t}^{\sharp} \\
\Omega_{t}^{b} & 0
\end{array}\right) .
$$

Here $\pi_{t}^{\sharp}: A^{*} \rightarrow A$ denotes the inverse of the bundle map $\Omega_{t}^{b}: A \rightarrow A^{*}$ associated to the symplectic form $\Omega_{t}$.

It is well known that

$$
\lambda_{t}: A_{\mathbb{C}} \rightarrow L_{t}: X \mapsto X-i \Omega_{t}^{b} X
$$

is a Lie algebroid isomorphism. Therefore, the diagram

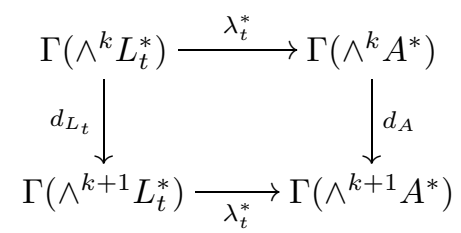

is commutative.

Observe that $\lambda_{t}^{*} \dot{\omega}_{t}=-\dot{\Omega}_{t}$. Indeed,

$$
\lambda_{t}^{*} \dot{\omega}_{t}(X, Y)=\left\langle\lambda_{t} X, \dot{J}_{t} \lambda_{t} Y\right\rangle=\left\langle\left(\begin{array}{c}
X \\
-i \Omega_{t}^{b} X
\end{array}\right),\left(\begin{array}{cc}
0 & -\dot{\pi}_{t}^{\sharp} \\
\dot{\Omega}_{t}^{b} & 0
\end{array}\right)\left(\begin{array}{c}
Y \\
-i \Omega_{t}^{b} Y
\end{array}\right)\right\rangle=-\dot{\Omega}_{t}(X, Y) .
$$

Here we have used the identity $\dot{\Omega}_{t}^{b}=-\Omega_{t}^{b} \circ \dot{\pi}_{t}^{\sharp} \circ \Omega_{t}^{b}$, which is easily obtained from $\pi_{t}^{\sharp} \circ \Omega_{t}^{b}=$ id. Hence

$$
d_{A} \dot{\Omega}_{t}=-d_{A} \lambda_{t}^{*} \dot{\omega}_{t}=-\lambda_{t}^{*} d_{L_{t}} \dot{\omega}_{t}=0
$$


Now assume there exists a family of smooth sections $\xi_{t} \in \Gamma\left(A^{*}\right)$ such that $\dot{\Omega}_{t}=$ $-d_{A} \xi_{t}$. It is easy to check that $\xi_{t}=\lambda_{t}^{*} \beta_{t}$, where $\beta_{t}=\left\langle\overline{\left\langle i \lambda_{t} \pi_{t}^{\sharp} \xi_{t}\right.}, \cdot\right\rangle$. Hence

$$
\lambda_{t}^{*} \dot{\omega}_{t}=-\dot{\Omega}_{t}=d_{A} \xi_{t}=d_{A} \lambda_{t}^{*} \beta_{t}=\lambda_{t}^{*} d_{L_{t}} \beta_{t}
$$

i.e., $\dot{\omega}_{t}=d_{L_{t}} \beta_{t}$.

By Theorem 3.4, we have $\phi_{t *} J_{t}=J_{0}$, where $\phi_{t}$ is the Courant algebroid automorphism generated by $\delta_{\pi_{t}^{\sharp} \xi_{t}}$. Since $\pi_{t}^{\sharp} \xi_{t} \in \Gamma(A), \phi_{t}$ is actually induced by a Lie algebroid automorphism of $A$, which will also be denoted by $\phi_{t}$ by abuse of notation.

We have proved the following Lie algebroid version of Moser's lemma [24].

Corollary 4.1. Assume that $M$ is a compact manifold and that $A$ is a Lie algebroid over $M$. Assume that $\Omega_{0}$ and $\Omega_{1} \in \Gamma\left(\wedge^{2} A^{*}\right)$ are two symplectic structures on the Lie algebroid $A$ which can be connected by a smooth family of symplectic structures $t \mapsto \Omega_{t}(t \in[0,1])$ on $A$. Assume there exists a smooth path $t \mapsto \xi_{t}$ in $\Gamma\left(A^{*}\right)$ such that $\dot{\Omega}_{t}=-d_{A} \xi_{t}, \forall t$. Then $\Omega_{0}$ and $\Omega_{1}$ are isomorphic. That is, there is a Lie algebroid automorphism $\phi$, isotopic to the identity, such that $\phi^{*} \Omega_{1}=\Omega_{0}$.

Example 2. Let $A \rightarrow M$ be a Lie algebroid endowed with an integrable almost complex structure, i.e. a bundle map $j: A \rightarrow A$, over the identity, whose Nijenhuis tensor vanishes and such that $j^{2}=-$ id. Its complexification $A_{\mathbb{C}}=A \otimes \mathbb{C}$ decomposes as the direct sum $A^{1,0} \oplus A^{0,1}$ of the eigenbundles of $j$ with eigenvalues $+i$ and $-i$, respectively. Both $A^{1,0}$ and $A^{0,1}$ are Lie subalgebroids of $A_{\mathbb{C}}$. The vector bundle $A^{1,0}$ is naturally an $A^{0,1}$-module: the representation of $A^{0,1}$ on $A^{1,0}$ is the map

$$
\Gamma\left(A^{0,1}\right) \times \Gamma\left(A^{1,0}\right) \rightarrow \Gamma\left(A^{1,0}\right):(X, Y) \mapsto X \triangleright Y:=\operatorname{pr}^{1,0}[X, Y]
$$

where $\operatorname{pr}^{1,0}$ stands for the canonical projection $A_{\mathbb{C}}=A^{0,1} \oplus A^{1,0} \rightarrow A^{1,0}$.

Now consider the dual vector bundle $A^{*} \rightarrow M$ as a trivial Lie algebroid; i.e., the anchor and the bracket are both zero. The pair $\left(A, A^{*}\right)$ is a Lie bialgebroid and the endomorphism

$$
J=\left(\begin{array}{cc}
-j & 0 \\
0 & j^{*}
\end{array}\right)
$$

is a generalized complex structure on its double $E:=A \oplus A^{*}$. The $+i$-eigenbundle $L$ of $J$ is naturally isomorphic, as a Lie algebroid, to the semi-direct product Lie algebroid $A^{0,1} \ltimes\left(A^{1,0}\right)^{*}$, where $\left(A^{1,0}\right)^{*}$ is the $A^{0,1}$-module dual to $A^{1,0}$.

The following lemma can be easily verified.

Lemma 4.2. Let $A$ be a Lie algebroid, let $M$ be an $A$-module and let $A \ltimes M^{*}$ be the resulting semi-direct product Lie algebroid. Then the diagram

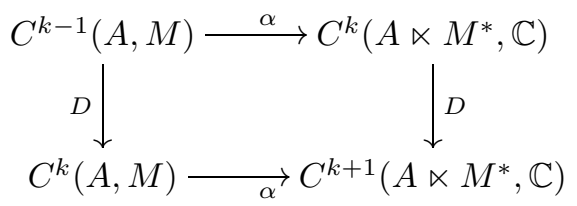

commutes for all $k \geq 0$. Here $C^{k}(A, M):=\Gamma\left(\wedge^{k} A^{*} \otimes M\right)$, the $D$ 's are the coboundary operators for the Lie algebroid cohomologies of $A$ (with values in the module $M)$ and $A \ltimes M^{*}$, while $\alpha$ is the skew-symmetrization map

$$
\left(\wedge^{k} A^{*}\right) \otimes M \rightarrow\left(\wedge^{k} A^{*}\right) \wedge M \hookrightarrow \wedge^{k+1}\left(A \ltimes M^{*}\right)^{*} .
$$


Let $t \mapsto j_{t}, t \in[0,1]$ be a smooth family of integrable almost complex structures on $A$. Let

$$
J_{t}=\left(\begin{array}{cc}
-j_{t} & 0 \\
0 & j_{t}^{*}
\end{array}\right)
$$

be the associated family of generalized complex structures on the Courant algebroid $E=A \oplus A^{*}$.

Taking $A=A_{t}^{0,1}$ and $M=A_{t}^{1,0}$ in Lemma 4.2, we get that

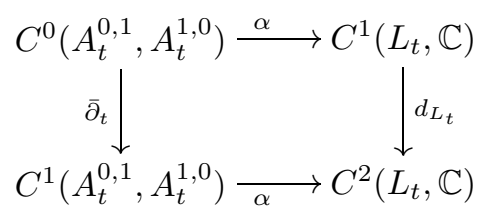

is commutative for all $t \in[0,1]$. Here $\bar{\partial}$ denotes the differential operator of the Lie algebroid cohomology of $A_{t}^{0,1}$ with values in the module $A_{t}^{1,0}$.

The time derivative $\dot{j}_{t}$ may be seen as an element of $\Gamma\left(\left(A_{t}^{0,1}\right)^{*} \otimes A_{t}^{1,0}\right)=$ $C^{1}\left(A_{t}^{0,1}, A_{t}^{1,0}\right)$ because $\dot{j}_{t}$ swaps $A_{t}^{0,1}$ and $A_{t}^{1,0}$. Moreover, $\dot{\omega}_{t}=-\alpha\left(\dot{j}_{t}\right)$. Indeed, for all $X, Y \in \Gamma\left(A_{t}^{0,1}\right)$ and all $\xi, \eta \in \Gamma\left(\left(A_{t}^{1,0}\right)^{*}\right)$, one has

$$
\begin{aligned}
\dot{\omega}_{t}(X+\xi, Y+\eta)=\left\langle X+\xi, \dot{J}_{t}(Y+\eta)\right\rangle=\left\langle X+\xi, \dot{j}_{t} Y\right. & \left.-\dot{j}_{t}^{*} \eta\right\rangle \\
= & \frac{1}{2}\left(\xi\left(\dot{j}_{t} Y\right)-\eta\left(\dot{j}_{t} X\right)\right)=-\alpha\left(\dot{j}_{t}\right)(X+\xi, Y+\eta) .
\end{aligned}
$$

Assume there exists a family $t \in[0,1] \mapsto z_{t} \in \Gamma\left(A_{t}^{0,1}\right)$ such that

$$
\bar{\partial}_{t}\left(\frac{1}{2} \overline{z_{t}}\right)=-\dot{j}_{t} .
$$

(Remark that $\frac{1}{2} \overline{z_{t}} \in \Gamma\left(A_{t}^{1,0}\right)=C^{0}\left(A_{t}^{0,1}, A_{t}^{1,0}\right)$.) Then

$$
\dot{\omega}_{t}=-\alpha\left(\dot{j}_{t}\right)=\alpha \circ \bar{\partial}_{t}\left(\frac{1}{2} \overline{z_{t}}\right)=d_{L_{t}} \circ \alpha\left(\frac{1}{2} \overline{z_{t}}\right)=d_{L_{t}}\left(\left\langle\overline{z_{t}}, \cdot\right\rangle\right),
$$

since $\left\langle\overline{z_{t}}, X+\xi\right\rangle=\frac{1}{2} \xi\left(\overline{z_{t}}\right)=\frac{1}{2} \alpha\left(\overline{z_{t}}\right)(\xi)$ for all $X+\xi \in L_{t}=A_{t}^{0,1} \ltimes\left(A_{t}^{1,0}\right)^{*}$.

Set $x_{t}=\frac{1}{2 i}\left(z_{t}-\overline{z_{t}}\right) \in \Gamma(A)$. According to Theorem 3.4 we have $\phi_{t *} J_{t}=J_{0}$, where $\phi_{t}$ is the flow of automorphisms of the Courant algebroid $E=A \oplus A^{*}$ generated by $\delta_{x_{t}}$. Here, since the section $x_{t}$ of $E$ actually lies in the Lie algebroid $A, \phi_{t}$ is induced by a family of Lie algebroid isomorphisms.

We have proved

Corollary 4.3. Let $A$ be a Lie algebroid over a compact manifold $M$. Let $j_{0}$ and $j_{1}$ be two integrable almost complex structures on A, which are connected by a smooth family $j_{t}$ of integrable almost complex structures. Assume there exists a smooth family $z_{t} \in \Gamma\left(A_{t}^{0,1}\right)$ such that $\bar{\partial}_{t} \overline{z_{t}}=-2 \dot{j}_{t}$. Then $j_{0}$ and $j_{1}$ are isomorphic; i.e., there exists a Lie algebroid automorphism $\phi$ such that $j_{1}=\phi_{*} j_{0}\left(:=\phi^{-1} \circ j_{0} \circ \phi\right)$.

When $A$ is the tangent Lie algebroid $T M$, this is a classical result [19].

\section{HAMiLtonian OPERATORS}

Let $J$ be a generalized complex structure on a Courant algebroid $E$. Then $E=L \oplus \bar{L}$ and $L^{*} \simeq \bar{L}$, where $L$ is the $+i$-eigenbundle of $J$ and $\left(L, L^{*}\right)$ is a complex Lie bialgebroid.

Modulo the isomorphism $E \rightarrow E^{*}: e \mapsto\langle e, \cdot\rangle$, which identifies $\bar{L}$ with $L^{*}$ (resp. $L$ with $\left.\bar{L}^{*}\right)$, any section $H \in \Gamma\left(\wedge^{2} L^{*}\right)\left(\right.$ resp. $\left.\bar{H} \in \Gamma\left(\wedge^{2} \bar{L}^{*}\right)\right)$ can be seen as a map 
$H^{\prime}: L \rightarrow \bar{L}\left(\right.$ resp. $\left.\bar{H}^{\prime}: \bar{L} \rightarrow L\right)$. The graph $L_{H}=\left\{\left(v, H^{\prime} v\right)\right\}_{v \in L} \subset L \oplus \bar{L}$ of $H^{\prime}: L \rightarrow \bar{L}$ is a Dirac structure of $E$ if, and only if, the Maurer-Cartan equation

$$
d_{L} H+\frac{1}{2}[H, H]_{L^{*}}=0
$$

holds [22]. In this case, $H$ is called a Hamiltonian operator. Here $d_{L}: \Gamma\left(\wedge^{\bullet} L^{*}\right) \rightarrow$ $\Gamma\left(\wedge^{\bullet+1} L^{*}\right)$ is the Lie algebroid differential of $L$ and $[\cdot, \cdot]_{L^{*}}: \Gamma\left(\wedge^{k} L^{*}\right) \otimes \Gamma\left(\wedge^{l} L^{*}\right) \rightarrow$ $\Gamma\left(\wedge^{k+l-1} L^{*}\right)$ is the Schouten bracket of the Lie algebroid $L^{*}$.

When $H$ is a Hamiltonian operator, $L_{H}$ is a Lie algebroid. The vector bundle isomorphism

$$
L \rightarrow L_{H}: v \mapsto\left(v, H^{\prime} v\right)
$$

induces the cochain complex isomorphism

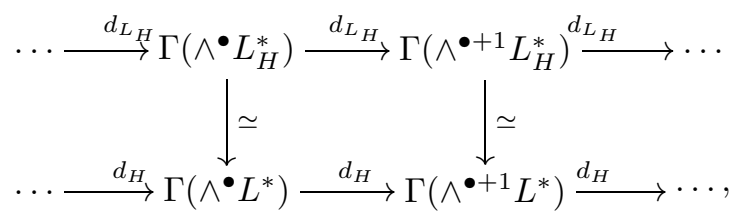

where $d_{H}=d_{L}+[H, \cdot]_{L^{*}}$ and $d_{L_{H}}$ is the Lie algebroid coboundary operator of $L_{H}$.

Lemma 5.1. Assume $H \in \Gamma\left(\wedge^{2} L^{*}\right)$ is a Hamiltonian operator. Then $L_{H}$ is the $+i$-eigenbundle of a generalized complex structure on $E$ if, and only if, the bundle map $\bar{H}^{\prime} \circ H^{\prime}-1: L \rightarrow L$ is invertible.

Proof. First, note that $\overline{\left(L_{H}\right)}=(\bar{L})_{\bar{H}}$. Hence $\left(v, H^{\prime} v\right) \in L_{H} \cap \overline{L_{H}}$ iff $\exists w \in \bar{L}$ such that $\left(v, H^{\prime} v\right)=\left(\bar{H}^{\prime} w, w\right)$, which holds if, and only if, $\left(\bar{H}^{\prime} \circ H^{\prime}-1\right) v=0$. It thus follows that $L_{H} \cap \overline{L_{H}}=\{0\}$ iff $\bar{H}^{\prime} \circ H^{\prime}-1$ is invertible.

Example 5.2. Consider the generalized complex structure $J=\left(\begin{array}{cc}-j & 0 \\ 0 & j^{*}\end{array}\right)$ on the standard Courant algebroid $E=T M \oplus T^{*} M$ corresponding to a complex structure $j: T M \rightarrow T M$. Then we have $L=T^{0,1} \ltimes\left(T^{1,0}\right)^{*}$ and $\bar{L}=T^{1,0} \ltimes\left(T^{0,1}\right)^{*}$, where the Lie algebroid structure on $L$ is the semi-direct product, similarly for $\bar{L}$. Let $\pi \in \Gamma\left(\wedge^{2} T^{1,0}\right)$. It is simple to see that $\bar{\pi}^{\prime} \circ \pi^{\prime}=0$. Hence $\bar{\pi}^{\prime} \circ \pi^{\prime}-1=-1$ is invertible. On the other hand, $\pi$ satisfies the Maurer-Cartan equation if and only if $\bar{\partial} \pi=0$ and $[\pi, \pi]=0$. That is, $\pi$ is a holomorphic Poisson structure. Thus we recover the well-known fact that a holomorphic Poisson structure is a generalized complex structure [13, 12] on the standard Courant algebroid $T M \oplus T^{*} M$.

Now, let $t \in[0,1] \mapsto H_{t} \in \Gamma\left(\wedge^{2} L^{*}\right)$ be a family of Hamiltonian operators such that $\overline{H_{t}} \circ \circ H_{t}^{\prime}-1$ is invertible for all $t \in[0,1]$. By Lemma 5.1, we obtain a family of generalized complex structures $t \mapsto J_{t}$. In this case, its corresponding $\dot{\omega}_{t}$ has a very simple description.

Lemma 5.3. $\Theta_{t}^{*} \dot{\omega}_{t}=-i \dot{H}_{t} \quad \in \Gamma\left(\wedge^{2} L^{*}\right)$.

Here $\Theta_{t}$ is the vector bundle map

$$
L \rightarrow E=L \oplus \bar{L}: v \mapsto\left(v, H_{t}^{\prime} v\right)
$$

identifying $L$ with $L_{H_{t}}$. 
Proof. By definition, we have

$$
J_{t}\left(v, H_{t}^{\prime} v\right)=i\left(v, H_{t}^{\prime} v\right) .
$$

Differentiating w.r.t. $t$, we get

$$
\dot{J}_{t}\left(v, H_{t}^{\prime} v\right)+J_{t}\left(0, \dot{H}_{t}^{\prime} v\right)=i\left(0, \dot{H}_{t}^{\prime} v\right) .
$$

It thus follows that

$$
\begin{aligned}
\Theta_{t}^{*} \dot{\omega}_{t}(v, w) & =\left\langle\left(v, H_{t}^{\prime} v\right), \dot{J}_{t}\left(w, H_{t}^{\prime} w\right)\right\rangle=\left\langle\left(v, H_{t}^{\prime} v\right),\left(i-J_{t}\right)\left(w, H_{t}^{\prime} w\right)\right\rangle \\
= & \left\langle\left(i+J_{t}\right)\left(v, H_{t}^{\prime} v\right),\left(w, H_{t}^{\prime} w\right)\right\rangle=2 i\left\langle\left(v, H_{t}^{\prime} v\right),\left(w, H_{t}^{\prime} w\right)\right\rangle=-i \dot{H}_{t}(v, w) .
\end{aligned}
$$

From (5.1) and Lemma 5.3, it follows that (3.3) is equivalent to

$$
\frac{d H_{t}}{d t}=d_{L} Y_{t}+\left[H_{t}, Y_{t}\right]_{L^{*}}
$$

where $Y_{t}=i \Theta_{t}^{*} \beta_{t} \in \Gamma\left(L^{*}\right)$. We have proved

Theorem 5.4. Let $E$ be a Courant algebroid over a compact manifold $M$ whose complexification $E_{\mathbb{C}}$ is the direct sum $L \oplus \bar{L}$ of a pair of complex conjugated complex Lie algebroids. Let $H_{t} \in \Gamma\left(\wedge^{2} L^{*}\right)$ be a smooth family of Hamiltonian operators such that $\overline{H_{t}} \circ H_{t}^{\prime}-1$ is invertible. Assume there exists a smooth family $Y_{t} \in \Gamma\left(L^{*}\right)$ solving the equation

$$
\frac{d H_{t}}{d t}=d_{L} Y_{t}+\left[H_{t}, Y_{t}\right]_{L^{*}}
$$

Then the generalized complex structures associated to $H_{0}$ and $H_{1}$ are isomorphic.

Example 5.5. Take $L=T^{0,1} \ltimes\left(T^{1,0}\right)^{*}$ as in Section 4, Let $\pi_{t} \in \Gamma\left(\wedge^{2} T^{1,0}\right)$ be a family of holomorphic Poisson bivector fields. It is simple to check that $Y_{t}=$ $X_{t}^{1,0}+\xi_{t}^{0,1} \in \bar{L}=T^{1,0} \ltimes\left(T^{0,1}\right)^{*}$ satisfies (5.2) iff

$$
\bar{\partial} X_{t}^{1,0}=0, \quad \bar{\partial} \xi_{t}^{0,1}=0 \quad \text { and } \quad \dot{\pi}_{t}=\left[\pi_{t}, X_{t}^{1,0}\right] .
$$

In particular, one may take $\xi_{t}^{0,1}=0$. Thus we obtain the following standard result: if there exists a family of complete holomorphic vector fields $X_{t}^{1,0}$ satisfying the equation

$$
\dot{\pi}_{t}=\left[\pi_{t}, X_{t}^{1,0}\right],
$$

then the holomorphic Poisson structures $\pi_{0}$ and $\pi_{1}$ are isomorphic. Note that, in this case, $x_{t}=2 \operatorname{Re} X_{t}^{1,0} \in \mathfrak{X}(M)$ and $x_{t}$ is complete iff $X_{t}^{1,0}$ is complete.

\section{Darboux-Weinstein theorem}

As an application of Theorem 3.4, we present a Darboux-Weinstein style theorem for generalized complex structures.

Let $J$ be a generalized complex structure on the standard Courant algebroid $T M \oplus T^{*} M \rightarrow M$. Then $\Gamma\left(T M \oplus T^{*} M\right)$ is a module over the ring $C^{\infty}(M)$. Given a point $o \in M$, choose local coordinates $\left(x_{1}, \ldots, x_{n}\right)$ identifying an open neighbourhood $U$ of $o$ in $M$ with a unit ball in $\mathbb{R}^{n}$ centered at the origin (and 
mapping $o$ to 0$)$. Then $\left\{e_{1}=\partial_{x^{1}}, \ldots, e_{n}=\partial_{x^{n}}, e_{n+1}=d x^{1}, \ldots, e_{2 n}=d x^{n}\right\}$ is a local frame of $T M \oplus T^{*} M$ over $U$, which enjoys the following remarkable properties:

$$
\begin{gathered}
\llbracket e_{i}, e_{j} \rrbracket=0, \quad \forall i, j \in\{1, \ldots, 2 n\} ; \\
\left\langle e_{i}, e_{j}\right\rangle \text { is constant on } U, \quad \forall i, j \in\{1, \ldots, 2 n\} .
\end{gathered}
$$

Now consider the matrix representation of $J$ in this local frame:

$$
\left.J e_{j}\right|_{x}=\left.\sum_{i} a_{j}^{i}(x) e_{i}\right|_{x}, \quad x \in U
$$

The $a_{j}^{i}$ 's are smooth functions on $U \subset \mathbb{R}^{n}$. Set

$$
\left.J_{t} e_{j}\right|_{x}=\left.\sum_{i} a_{j}^{i}(t x) e_{i}\right|_{x}
$$

for all $t \in[0,1]$ and $x \in U$. Clearly, $J_{t}^{2}=-\mathrm{id}, J_{1}=J$ and $J_{0}$ is represented by the constant matrix $a_{j}^{i}(0)$. Moreover, (6.1) implies that $J_{0}$ is integrable.

Lemma 6.1. All $J_{t}$ 's are generalized complex structures on the restriction of $T M \oplus$ $T^{*} M$ to $U$.

Proof. Let $\left\{e^{k}\right\}_{k=1, \ldots, 2 n}$ be the dual frame to $\left\{e_{i}\right\}_{i=1, \ldots, 2 n}$. From a tedious computation using the relations (6.1), (6.2) and

$$
\llbracket f A, g B \rrbracket=f g \llbracket A, B \rrbracket+f(\rho(A) g) B-g(\rho(B) f) A+\langle A, B\rangle(g \mathcal{D} f-f \mathcal{D} g)
$$

(where $f, g \in C^{\infty}(M)$ and $A, B \in \Gamma\left(T M \oplus T^{*} M\right)$ ), it follows that

$$
\begin{gathered}
\left(e^{k}, \llbracket J_{t} e_{i}, J_{t} e_{j} \rrbracket\right)(x)=t\left(e^{k}, \llbracket J e_{i}, J e_{j} \rrbracket\right)(t x), \\
\left(e^{k}, \llbracket J_{t} e_{i}, e_{j} \rrbracket\right)(x)=t\left(e^{k}, \llbracket J e_{i}, e_{j} \rrbracket\right)(t x),
\end{gathered}
$$

and

$$
\left(e^{k}, J_{t} \llbracket J_{t} e_{i}, e_{j} \rrbracket\right)(x)=t\left(e^{k}, J \llbracket J e_{i}, e_{j} \rrbracket\right)(t x) .
$$

Hence the integrability of $J$ at the point $t x$ implies the integrability of $J_{t}$ at the point $x$. Since $J$ is integrable on $U, U$ is the unit ball around 0 in $\mathbb{R}^{n}$ and $t \in[0,1]$, the conclusion follows.

As an immediate consequence of Theorem 3.4. we have

Corollary 6.2. Assume there exists a smooth path $t \in[0,1] \mapsto \beta_{t} \in \Gamma\left(\left.L_{t}^{*}\right|_{U}\right)$ such that $\dot{\omega}_{t}=d_{L_{t}} \beta_{t}$. Then the restriction of $J$ to $U$ is isomorphic to the constant generalized complex structure $J_{0}$.

Theorem 6.3. Let $J$ be a generalized complex structure (with $+i$-eigenbundle $L$ ) on the standard Courant algebroid $T M \oplus T^{*} M \rightarrow M$. Let $\left(x_{1}, \ldots, x_{n}\right)$ be local coordinates on $M$ identifying an open domain $U$ with the unit ball centered at the origin in $\mathbb{R}^{n}$ and define $J_{t}$ as in (6.3). If there exists $\beta \in \Gamma\left(\left.L^{*}\right|_{U}\right)$ such that

$$
d_{L} \beta=\dot{\omega}_{1},\left.\quad \beta\right|_{0}=0 \text { and }\left.d \beta\right|_{0}=0,
$$

then $\left.J\right|_{U}$ is isomorphic to a constant generalized complex structure.

Remark 6.4. Since, at a singular point (for the underlying Poisson structure [2, 4, 5, 17, 8, 9, 10, 13, 14, 15, 16]), a generalized complex structure cannot be isomorphic to a constant one, the condition of Theorem 6.3 must fail in any neighbourhood of any singular point. 
The proof is split into several lemmas.

Lemma 6.5. Let $E_{1}$ and $E_{2}$ be two Courant algebroids over the same base manifold $M$, with anchor maps $\rho_{1}$ and $\rho_{2}$, respectively. Let

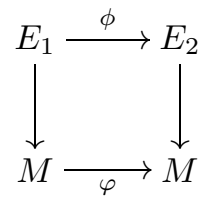

be an isomorphism of vector bundles such that

$$
\rho_{2} \circ \phi=\varphi_{*} \circ \rho_{1}
$$

and

$$
\llbracket \phi^{-1} \circ \alpha \circ \varphi, \phi^{-1} \circ \beta \circ \varphi \rrbracket=\phi^{-1} \circ \llbracket \alpha, \beta \rrbracket \circ \varphi, \quad \forall \alpha, \beta \in \Gamma\left(E_{2}\right) .
$$

Then $\phi$ establishes a 1-1 correspondence between the subbundles of $E_{1}$ and $E_{2}$ which are Lie algebroids.

Lemma 6.6. The vector bundle map

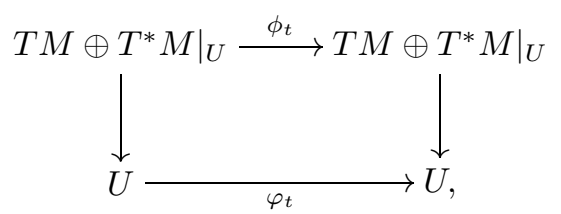

where

$$
\varphi_{t}\left(x_{1}, \ldots, x_{n}\right)=\left(t x_{1}, \ldots, t x_{n}\right)
$$

and

$$
\phi_{t}\left(\left.e_{i}\right|_{x}\right)=\left.t e_{i}\right|_{t x}
$$

satisfies the assumptions of Lemma 6.5. Moreover, we have

$$
J \circ \phi_{t}=\phi_{t} \circ J_{t} .
$$

Corollary 6.7. The "multiplication by t" map

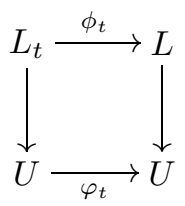

is a Lie algebroid isomorphism.

Therefore we have

$$
\phi_{t}^{*} \circ d_{L}=d_{L_{t}} \circ \phi_{t}^{*}
$$

Lemma 6.8.

$$
t^{3} \dot{\omega}_{t}=\phi_{t}^{*} \dot{\omega}_{1}
$$


Proof. Differentiating (6.3) w.r.t. $t$, we get

$$
\left.\dot{J}_{t} e_{j}\right|_{x}=\left.\left.\sum_{k, l} x^{l} \frac{\partial a_{j}^{k}}{\partial x^{l}}\right|_{t x} e_{k}\right|_{x} .
$$

Therefore

$$
\dot{\omega}_{t}\left(\left.e_{i}\right|_{x},\left.e_{j}\right|_{x}\right)=\left\langle\left. e_{i}\right|_{x},\left.\dot{J}_{t} e_{j}\right|_{x}\right\rangle=\left.b_{i j} x^{l} \frac{\partial a_{j}^{k}}{\partial x^{l}}\right|_{t x} .
$$

Substituting 1 for $t$ and $t x$ for $x$ in the last relation, one gets

$$
\dot{\omega}_{1}\left(\left.e_{i}\right|_{t x},\left.e_{j}\right|_{t x}\right)=\left.b_{i j} t x^{l} \frac{\partial a_{j}^{k}}{\partial x^{l}}\right|_{t x} .
$$

Now, multiplying by $t^{2}$, we obtain

$$
\dot{\omega}_{1}\left(\left.t e_{i}\right|_{t x},\left.t e_{j}\right|_{t x}\right)=\left.b_{i j} t^{3} x^{l} \frac{\partial a_{j}^{k}}{\partial x^{l}}\right|_{t x}
$$

or, equivalently,

$$
\dot{\omega}_{1}\left(\left.\phi_{t} e_{i}\right|_{x},\left.\phi_{t} e_{j}\right|_{x}\right)=t^{3} \dot{\omega}_{t}\left(\left.e_{i}\right|_{x},\left.e_{j}\right|_{x}\right) .
$$

Proof of Theorem 6.3. According to Lemma 6.8,

$$
t^{3} \dot{\omega}_{t}=\phi_{t}^{*} \dot{\omega}_{1}=\phi_{t}^{*} d_{L} \beta=d_{L_{t}} \phi_{t}^{*} \beta .
$$

Now set $\beta_{t}=t^{-3} \phi_{t}^{*} \beta$. We have $\beta_{t}\left(\left.e_{i}\right|_{x}\right)=t^{-3} \beta\left(\left.\phi_{t} e_{i}\right|_{x}\right)=t^{-2} \beta\left(\left.e_{i}\right|_{t x}\right)$, which shows that $\beta_{t}$ is well defined for $t=0$ since $\left.\beta\right|_{0}=0$ and $\left.d \beta\right|_{0}=0$. The conclusion follows from Theorem 3.4 since $\dot{\omega}_{t}=d_{L_{t}} \beta_{t}$.

Remark 6.9. When $J$ is the generalized complex structure corresponding to a symplectic structure, it is straightforward to check that the Poincare lemma for de Rham cohomology ensures that the conditions of Theorem 6.3 hold at any point $m \in M$. As a consequence, we recover the Darboux-Weinstein theorem. However the one-parameter family of generalized complex structures (or family of symplectic structures) considered here is different from the one used in [29].

On the other hand, when $J$ is the generalized complex structure corresponding to an integrable complex structure $j: T M \rightarrow T M$, Kohn gave a proof of the Newlander-Nirenberg theorem using exactly the same $j_{t}$ as ours 20. Kohn's essential idea and major achievement was to prove the Poincaré lemma for Dolbeault cohomology using the description of this cohomology in terms of the integrable almost complex structure rather than holomorphic charts. As a consequence, he was able to deform the local chart used to define the family of local almost complex structures $t \mapsto j_{t}$ joining the given $j$ to the constant $j_{0}$ into a local chart in which $j$ is constant. It would be interesting to compare our condition with the one in 20 in that case.

Remark 6.10. Note that the degree 2 Lie algebroid cohomology of $L$ may not always be locally trivial for generic generalized complex structures even though the Poincaré lemma holds for both the symplectic and complex cases. For instance, when $J$ corresponds to a holomorphic Poisson structure $\pi$, the Lie algebroid cohomology of $L$ is simply the Poisson cohomology of $\pi$ [21], which may not always be locally trivial. 
On the other hand, the condition of Theorem 6.3 is stronger than just requiring $H^{2}\left(\left.L\right|_{U}, \mathbb{C}\right)$ to vanish locally on a neighbourhood $U$. For instance, when $J$ is the generalized complex structure corresponding to the holomorphic Lie Poisson structure of a complex simple Lie algebra, $H^{2}\left(\left.L\right|_{U}, \mathbb{C}\right)$ vanishes on a neighbourhood $U$ of 0 . However $J$ cannot be locally trivial on neighbourhoods of 0 .

\section{ACKNOWLEDGEMENTS}

The author is grateful to Alan Weinstein for the encouraging discussion they had in Berkeley at the early stage of this project and to Alberto Cattaneo, Vasiliy Dolgushev, Giovanni Felder, Camille Laurent-Gengoux, Pierre Schapira, Boris Tsygan, Aissa Wade and Ping Xu for other ones; he also thanks the E.S.I. Vienna and I.H.P. Paris for their hospitality while parts of this paper were written.

\section{REFERENCES}

1. Ralph Abraham and Jerrold E. Marsden, Foundations of mechanics, Benjamin/Cummings Publishing Co. Inc. Advanced Book Program, Reading, Mass., 1978, Second edition, revised and enlarged, With the assistance of Tudor Raţiu and Richard Cushman. MR515141 (81e:58025)

2. Vestislav Apostolov and Marco Gualtieri, Generalized Kähler manifolds, commuting complex structures, and split tangent bundles, Comm. Math. Phys. 271 (2007), no. 2, 561-575. MR.2287917

3. James Barton, Generalized complex structures on Courant algebroids, Ph.D. thesis, 2007.

4. Henrique Bursztyn, Gil R. Cavalcanti, and Marco Gualtieri, Generalized Kähler and hyperKähler quotients, Poisson geometry in mathematics and physics, 61-77, Contemp. Math., 450, Amer. Math. Soc., Providence, RI, 2008. MR 2397619 (2010e:53139)

5. _ Reduction of Courant algebroids and generalized complex structures, Adv. Math. 211 (2007), no. 2, 726-765. MR2323543 (2009d:53124)

6. Ana Cannas da Silva and Alan Weinstein, Geometric models for noncommutative algebras, Berkeley Mathematics Lecture Notes, vol. 10, American Mathematical Society, Providence, RI, 1999. MR1747916 (2001m:58013)

7. Gil R. Cavalcanti, Formality in generalized Kähler geometry, Topology Appl. 154 (2007), no. 6, 1119-1125. MR 2298627 (2008c:53076)

8. New aspects of the ddc-lemma. arXiv:math/0501406

9. Gil R. Cavalcanti and Marco Gualtieri, Generalized complex structures on nilmanifolds, J. Symplectic Geom. 2 (2004), no. 3, 393-410. MR2131642 (2006b:53098)

10. _ A surgery for generalized complex structures on 4-manifolds, J. Differential Geom. 76 (2007), no. 1, 35-43. MR 2312048

11. Theodore James Courant, Dirac manifolds, Trans. Amer. Math. Soc. 319 (1990), no. 2, 631661. MR998124 (90m:58065)

12. Marius Crainic, Generalized complex structures and Lie brackets. arXiv:math/0412097

13. Marco Gualtieri, Generalized complex geometry, DPhil thesis, 2003, arXiv:math/0401221

14. , Generalized complex geometry. arXiv:math/0703298

15. Generalized geometry and the Hodge decomposition. arXiv:math/0409093

16. Nigel Hitchin, Generalized Calabi-Yau manifolds, Q. J. Math. 54 (2003), no. 3, 281-308. MR2013140 (2004h:32024)

17. Shengda $\mathrm{Hu}$, Hamiltonian symmetries and reduction in generalized geometry, Houston J. Math. 35 (2009), no. 3, 787-811. MR2534281

18. _ Reduction and duality in generalized geometry, J. Symplectic Geom. 5 (2007), no. 4, 439-473. MR2413310 (2009m:53217)

19. Kunihiko Kodaira, Complex manifolds and deformation of complex structures, English ed., Classics in Mathematics, Springer-Verlag, Berlin, 2005, Translated from the 1981 Japanese original by Kazuo Akao. MR2109686 (2005h:32030)

20. J. J. Kohn, Harmonic integrals on strongly pseudo-convex manifolds. I, Ann. of Math. (2) 78 (1963), 112-148. MR0153030(27:2999) 
21. Camille Laurent-Gengoux, Mathieu Stiénon, and Ping Xu, Holomorphic Poisson manifolds and holomorphic Lie algebroids, Int. Math. Res. Not. IMRN 2008, Art. ID rnn 088, 46 pp. MR2439547 (2009i:53082)

22. Zhang-Ju Liu, Alan Weinstein, and Ping Xu, Manin triples for Lie bialgebroids, J. Differential Geom. 45 (1997), no. 3, 547-574. MR1472888 (98f:58203)

23. Kirill C. H. Mackenzie and Ping Xu, Lie bialgebroids and Poisson groupoids, Duke Math. J. 73 (1994), no. 2, 415-452. MR1262213 (95b:58171)

24. Jürgen Moser, On the volume elements on a manifold, Trans. Amer. Math. Soc. 120 (1965), 286-294. MR0182927 (32:409)

25. Ryszard Nest and Boris Tsygan, Deformations of symplectic Lie algebroids, deformations of holomorphic symplectic structures, and index theorems, Asian J. Math. 5 (2001), no. 4, 599-635. MR1913813 (2003k:53126)

26. A. Newlander and L. Nirenberg, Complex analytic coordinates in almost complex manifolds, Ann. of Math. (2) 65 (1957), 391-404. MR0088770 (19:577a)

27. Dmitry Roytenberg, Courant algebroids, derived brackets and even symplectic supermanifolds, Ph.D. thesis, 1999. arXiv:math/9910078

28. Pavol Ševera, Unpublished Letter to Alan Weinstein.

29. Alan Weinstein, Symplectic manifolds and their Lagrangian submanifolds, Advances in Math. 6 (1971), 329-346 (1971). MR0286137 (44:3351)

Departement Mathematik, E.T.H. Zürich, 8092 Zürich, Switzerland

E-mail address: stienon@math.ethz.ch

Current address: Institut de Mathématiques de Jussieu, Université Paris-Diderot, 75013 Paris, France

E-mail address: stienon@math.jussieu.fr 